\title{
CBMOS: a GPU-enabled Python framework for the numerical study of center-based models
}

\author{
Sonja Mathias* ${ }^{*}$, Adrien Coulier and Andreas Hellander
}

\section{${ }^{*}$ Correspondence:}

sonja.mathias@it.uu.se Department of Information Technology, Uppsala University, Uppsala, Sweden

\begin{abstract}
Background: Cell-based models are becoming increasingly popular for applications in developmental biology. However, the impact of numerical choices on the accuracy and efficiency of the simulation of these models is rarely meticulously tested. Without concrete studies to differentiate between solid model conclusions and numerical artifacts, modelers are at risk of being misled by their experiments' results. Most cell-based modeling frameworks offer a feature-rich environment, providing a wide range of biological components, but are less suitable for numerical studies. There is thus a need for software specifically targeted at this use case.
\end{abstract}

Results: We present CBMOS, a Python framework for the simulation of the centerbased or cell-centered model. Contrary to other implementations, CBMOS' focus is on facilitating numerical study of center-based models by providing access to multiple ordinary differential equation solvers and force functions through a flexible, userfriendly interface and by enabling rapid testing through graphics processing unit (GPU) acceleration. We show-case its potential by illustrating two common workflows: (1) comparison of the numerical properties of two solvers within a Jupyter notebook and (2) measuring average wall times of both solvers on a high performance computing cluster. More specifically, we confirm that although for moderate accuracy levels the backward Euler method allows for larger time step sizes than the commonly used forward Euler method, its additional computational cost due to being an implicit method prohibits its use for practical test cases.

Conclusions: CBMOS is a flexible, easy-to-use Python implementation of the centerbased model, exposing both basic model assumptions and numerical components to the user. It is available on GitHub and PyPI under an MIT license. CBMOS allows for fast prototyping on a central processing unit for small systems through the use of NumPy. Using CuPy on a GPU, cell populations of up to 10,000 cells can be simulated within a few seconds. As such, it will substantially lower the time investment for any modeler to check the crucial assumption that model conclusions are independent of numerical issues.

Keywords: Cell-based model, Center-based model, Numerical method, Implicit solver, Python, NumPy, CuPy author(s) and the source, provide a link to the Creative Commons licence, and indicate if changes were made. The images or other third party material in this article are included in the article's Creative Commons licence, unless indicated otherwise in a credit line to the material. If material is not included in the article's Creative Commons licence and your intended use is not permitted by statutory regulation or exceeds the permitted use, you will need to obtain permission directly from the copyright holder. To view a copy of this licence, visit http:// creativecommons.org/licenses/by/4.0/. The Creative Commons Public Domain Dedication waiver (http://creativecommons.org/publi cdomain/zero/1.0/) applies to the data made available in this article, unless otherwise stated in a credit line to the data. 


\section{Background}

Cell-based models offer the possibility to study questions relating cellular to population level behavior by explicitly representing each individual cell and its mechanical interaction with its neighbors [1]. As such, they are becoming increasingly popular for applications in developmental $[2,3]$ and cancer biology $[4,5]$.

There exist a multitude of cell-based models which can be categorized as either on- or off-lattice models. On-lattice models such as cellular automata [6] or the cellular Potts model [7] restrict the movement of cells in space to a fixed grid. Off-lattice models, on the other hand, track the movement of particles in continuous space. Depending on the resolution of the shape of the cells, the particles can represent cell midpoints (centerbased or cell-centered model [8,9]), cell membrane junctions (vertex-based model [10]) or even individual cell parts, such as in the immersed boundary method [11] or the subcellular element model [12]. Center-based models represent cells as overlapping spheres or as Voronoi polyhedra defined by the topology of their midpoints $[9,13]$. Cells then interact mechanically with their neighbors according to pairwise forces, in an analogy to them being connected by springs. The exact definition of the cell's neighborhood depends on the method, where the most common ones are either solely distance-based or restrict interaction to neighboring Voronoi polyhedra. Pairs of cells attract or repel each other depending on the distance between them. Currently available software for the general simulation of center-based models include the open-source frameworks Chaste [14], MecaGen [15], PhysiCell [16], and ya\|la [17], as well as the closed-source packages CellSys [18], EPISIM [19] and Biocellion [20].

Aside from relying on the basic modeling assumptions described above, the centerbased model includes many numerical aspects such as the method used to solve the system of ordinary differential equations (ODE) governing the movement of the cell midpoints. All open-source software implementations mentioned above use explicit low order integration methods, with Chaste and MecaGen using the most simple first-order forward Euler method [21], PhysiCell using the second-order Adams-Bashforth method [21] and lastly, yal|a implementing Heun's method [22], another second-order method. Contrary to other areas in the field of systems biology where the benefits of higher order and adaptive integration algorithms have been clearly established, this question has yet to be studied extensively in the case of cell-based models. It is our hope that providing a software designed specifically for ease-of-experimentation with different solvers will help shed empirical light on this issue.

For now, choosing all model components and their parameters - such as cutoff radii and spring stiffness values for the force functions or time step sizes for the numerical solver - as well as understanding their effects on the accuracy and efficiency of the simulation of population-level behavior remain open problems. Nevertheless, the robustness of biological conclusions to both basic model assumptions and numerical issues is of the utmost importance in order to build upon predictions and understanding gained from simulations. More specifically, with the increased usage of cell-based models there is an increased need for the numerical study of these models for several reasons [23]: (1) In the absence of exact data on intercellular forces in cell populations such as tissues, models abstract how cells mechanically interact with their immediate neighbors in different ways, e.g. with different types of ad-hoc pairwise interaction forces. Modelers need to 
study how their model conclusions at the population level are affected by changes to the exact mathematical definition of these force functions. (2) In general, it is not straightforward to know whether more complex numerical methods for solving the update equations-e.g. a higher order method or an implicit method-are beneficial over more simpler ones in terms of computational cost for a given level of accuracy. (3) Careful consideration needs to be given to the choice of purely numerical parameters, such as time step length, as these have been shown to affect model conclusions drawn from cellbased models [24].

Several publications have studied these three points in the context of center-based models. Such publications include the work of Pathmanathan et al. [25], where they compared the bulk mechanical properties of a non-proliferating tissue simulated using different physics-based forces, and the work by Atwell [26] which investigated both the use of different forces and of different numerical solvers for the simulation of a tumor growth type experiment. In particular, the latter compared a fourth-order explicit Runge-Kutta method and two implicit methods to the commonly used first-order explicit forward Euler method in terms of accuracy and run times, as well as proposing a simple adaptive mechanism to ensure that the time step size is chosen small enough to not violate a given absolute movement threshold on the displacement of the cell centers. Additionally, in [13] Osborne et al. compared five different cell-based models including the center-based model with respect to their underlying model assumptions, implementation details and applicability to different common biological problems. All of the above studies were done using the Chaste simulation framework. Furthermore, as part of the supplementary information of the publication announcing the PhysiCell code [16], Ghaffarizadeh et al. ran convergence studies for the second-order explicit Adams-Bashforth method (used by PhysiCell for updating the cell positions) for a two-cell test case and a compressed spheroid population.

In a previous study [27] we explored the question of how the formulation of the force function governing the pairwise interaction forces affects the numerical properties of the two-dimensional center-based model when used in combination with the different first and second-order explicit numerical methods commonly used in center-based model implementations. We showed that, for the simulation to remain physically correct, the size of the time step must be carefully tailored to the choice of force function. Moreover, choosing the time step size too large for a given force function/solver combination led to geometrical differences at the population level, with the different force functions exhibiting varying sensitivity to this issue. These findings illustrate the importance of ensuring that model conclusions are independent of numerical choices.

With the exception of [27], all of the above studies have been performed within a typical feature-rich modeling software written primarily to study biological problems within the context of a specific modeling problem. These frameworks typically only provide one type of force function and one solver and do not expose the numerical components of the model to the user (note that Chaste provides general interfaces which the user could extend [14]). As a complementary approach, there is value in studying these issues in a more general setting in order to inform modelers on how the combination of different basic model assumptions can affect typical population level behavior and how to avoid common pitfalls with respect to numerical parameters. 
To this end, we have written CBMOS, a framework designed explicitly for the numerical study of center-based models in two and three space dimensions. Our code is a Python implementation making it easily accessible for novice and experienced programmers alike, while internally relying heavily on NumPy's vectorized routines [28] for performance. Through the optional use of the CuPy library [29], it enables transfer of the calculation of the pairwise cellular forces to a graphics processing unit (GPU) if available, thus allowing for the simulation of cell population sizes of up to 10,000 cells in a few seconds.

CBMOS is publicly available on GitHub [30] along with all Jupyter notebooks that were used to generate the figures in this publication. Additionally, it is available on the Python Package Index (PyPI) and can be installed via pip by running pip install cbmos. It has minimal requirements consisting mainly of the scientific Python stack NumPy and SciPy, along with the optional requirement of CuPy if execution on the GPU is desired. Documentation can be found on the project's GitHub page [31]. Users interested at trying out CBMOS without installing it on their system, can also choose to run it through Google Colaboratory (or Google Colab for short) [32]. Google Colab offers a Python computing environment based on Jupyter notebooks with access to GPU hardware. It runs entirely in the cloud and is accessible through a web browser [33]. Most conveniently, it also features the possibility to directly run Jupyter notebooks hosted on GitHub, such as those that can be found in the CBMOS directory, enabling any potential user to quickly get started with CBMOS.

\section{Implementation}

In this section we briefly state the mathematical description of the center-based model as it is implemented in CBMOS and explain its design using a minimal working example. Moreover, we describe implementation aspects such as the array programming paradigm we use, as well as which parts of the code are extended to the GPU. Finally, we describe how interested users can install it.

\section{Mathematical description of the center-based model}

The center-based model implemented in CBMOS tracks the movements of the midpoint coordinates $\mathbf{x}$ of a population of cells over time. Individual cells are implicitly represented as circles (in two dimensions) or spheres (in three dimensions) with a fixed radius $R$. They are assumed to interact mechanically according to pairwise interactions with their neighbors within a certain maximum interaction distance $r_{A}$, with the magnitude of their force interaction depending only on their distance $r$. If two neighboring cells are located closer than some rest length $s$, they exert repulsive forces on each other to eliminate their overlap. If they are placed exactly the rest length $s$ apart, they exert no forces on each other. (As a default, CBMOS uses a rest length of $s=1.0$ cell diameter.) Additionally, if located in close proximity, but not overlapping (i.e. at a distance larger than the rest length $s$ but smaller than the maximum interaction distance $r_{A}$ ), they exert adhesive forces pulling them closer. Specific pairwise force functions implementing such behavior include the cubic force implemented in MecaGen [15], 


$$
F^{\text {cubic }}(r)= \begin{cases}\mu\left(r-r_{A}\right)^{2}(r-s) & \text { if } r \leq r_{A} \\ 0 & \text { otherwise }\end{cases}
$$

the piecewise quadratic (PWQ) force used in PhysiCell [16],

$$
F^{\mathrm{PWQ}}(r)= \begin{cases}\mu_{A}\left(1-\frac{r}{r_{A}}\right)^{2}-\mu_{R}\left(1-\frac{r}{r_{R}}\right)^{2} & \text { if } r \leq r_{R}, \\ \mu_{A}\left(1-\frac{r}{r_{A}}\right)^{2} & \text { if } r_{R}<r \leq r_{A}, \\ 0 & \text { otherwise, }\end{cases}
$$

and the generalized linear spring (GLS) force used in Chaste [14],

$$
F^{\mathrm{GLS}}(r)= \begin{cases}\mu \log (1+(r-s)) & \text { if } r \leq s \\ \mu(r-s) \exp (-\alpha(r-s)) & \text { if } s<r \leq r_{A} \\ 0 & \text { otherwise }\end{cases}
$$

In all three functions $\mu$ denotes the spring stiffness (split between repulsive and adhesive interactions for the piecewise quadratic force into the parameters $\mu_{R}$ and $\mu_{A}$ ). Furthermore, $r_{R}$ denotes the interaction distance for repulsive interactions for the piecewise quadratic force and $\alpha$ controls the width of the exponential decay of the GLS force in the adhesive regime (see [27] for an in-depth discussion and other force function examples). These one-dimensional forces are extended to two or three dimensions by multiplication with the normalized direction vector between cell midpoints, i.e. we define the pairwise force vector between cells $i$ and $j$ as $\mathbf{F}_{i j}=F\left(\left\|\mathbf{r}_{i j}\right\|\right) \frac{\mathbf{r}_{i j}}{\left\|\mathbf{r}_{i j}\right\|}$, where $\mathbf{r}_{i j}=\mathbf{x}^{(j)}-\mathbf{x}^{(i)}$ and $\mathbf{x}^{(i)}$ and $\mathbf{x}^{(j)}$ denote the midpoint coordinates of cells $i$ and $j$. Other force functions implemented in CBMOS include the linear force [34, 35] and the Hertz force [36, 37].

The cells are assumed to move in a microenvironment with a very low Reynolds number [38] in which inertial effects can be neglected. Under this assumption, the update equation for the midpoint coordinates of the $i$ th cell is

$$
-\eta \dot{\mathbf{x}}^{(i)}=\sum_{j \neq i} \mathbf{F}_{i j}
$$

where the drag force proportional to the cell's velocity is balanced with the force being exerted on the cell by its neighbors. The drag coefficient $\eta$ acts as a scaling of the pairwise interaction forces and can thus be arbitrarily fixed as $\eta=1$.

Given an initial placement for the coordinates of the population, Eq. (4) is solved numerically at discrete time points. Methods implemented in CBMOS for doing this include the explicit first-order forward Euler method [21] (commonly used by other center-based model simulation software such as Chaste [14] and MecaGen [15]), the explicit second-order Midpoint [22], Heuns [22] (used by yal|a [17]) and Adams-Bashforth [21] methods (the latter is used by PhysiCell [16]), as well as the implicit first-order backward Euler method [21]. Moreover, it is also easily possible to use other solvers from the standard library thanks to CBMOS being compatible with scipy. integrate's interface that provides access to several high-order numerical methods as well as LSODA [39]. We refer to the results section for a detailed description and comparison of the forward and backward Euler methods. 


\section{Design overview}

CBMOS allows to probe the interplay of different model components-mainly the pairwise interaction force and the numerical solver-and their combined effect on the population level behavior as well as on the efficiency of the simulation. To do so, CBMOS provides a flexible, easy-to-use interface that is easily expandable to study the effects of force functions, ODE solvers, time step sizes or cellular events in the context of centerbased models. CBMOS implements a number of pairwise force functions found in the literature and other popular software packages for the simulation of center-based models, as well as five ODE solvers, including three second-order solvers and one implicit solver. A simple example showing how to set up and run a simulation of two cells can be seen in Listing 1, with a more complex example being described on CBMOS' documentation web page [31]. Furthermore, the interested user can find code examples on common numerical analysis workflow scenarios in the Jupyter notebooks available in the GitHub repository (see the examples folder, as well as the code belonging specifically to this and our previous publication [27]).

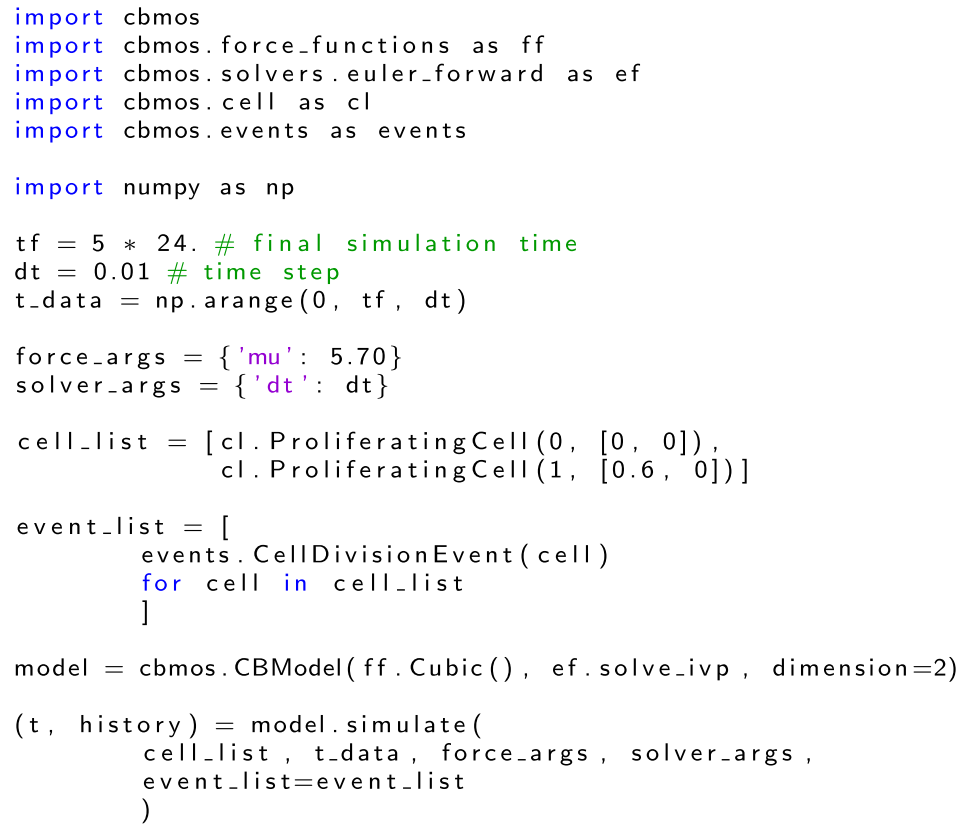

The CBMOS code is event-driven, meaning that cell events are queued according to their execution time and the mechanical equations for the center positions are solved in between the execution of cell events, see Fig. 1 for the general structure of the code. The event-driven implementation is advantageous for the numerical study of center-based models as it avoids an additional splitting error that arises when simulations proceed in a step-driven manner. Nevertheless, we allow for cellular events to be aggregated at fixed time points to improve simulation efficiency when a large number of cellular events need to be handled, as is commonly done in major modeling software. For those experiments described in the results and discussion section that include proliferation (i.e. the monolayer growth benchmark and the small monolayer population undergoing intense proliferation), we used simple cell division events where division times were determined 


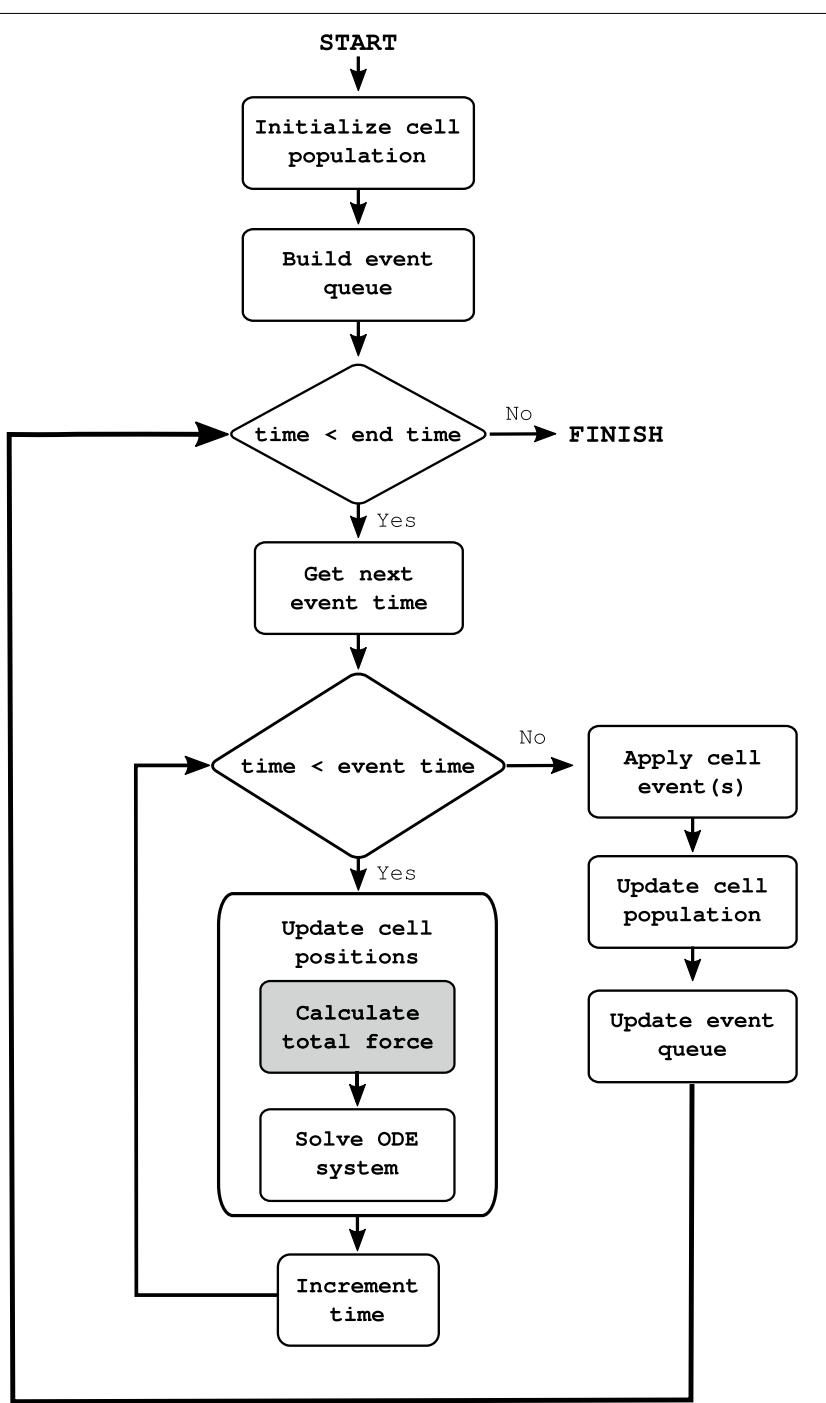

Fig. 1 Simplified flow chart for the CBMOS code. The positions of the cell midpoints are calculated numerically with the ODE solver between any two consecutive cell events. At that point, the next event is resolved and new events are queued. This process is repeated until the end time is reached. If the aggregation of cellular events is needed for efficiency, the event queue is built with event times rounded to the next possible event time according to the desired resolution. The main bottleneck consists of the calculation of the total force, highlighted with a darker background. This is where CuPy is used as a drop-in replacement for NumPy when access to a GPU is available

at cell birth. However, CBMOS' interface allows for the implementation of a variety of cellular events. Examples illustrating cell cycles that depend on the cellular environment, and cell death, can be found in the package's documentation.

\section{The use of array programming and extension to the GPU}

Simple center-based model implementations typically target cell counts from a few hundred to a few thousand cells. Larger system sizes are possible by using parallel implementations in a compiled language as done e.g. by PhysiCell [16], Chaste [40] or Biocellion [20]. Additionally, the yalla framework [17] achieves high performance by executing 
CUDA/C ++ code on GPUs. We follow a complementary approach in our code as its main purpose lies in facilitating the numerical study of the model components. As such, performance is secondary to fast prototyping, but nevertheless the possibility of simulating more cells in less time would allow for more realistic test problems and is thus very desirable. The three main points of our approach can be summarized as (i) the use of the Python programming language to enable fast prototyping [41], (ii) the use of array programming via NumPy to achieve a reasonable performance on the central processing unit (CPU) and (iii) the use of a high-level GPU-library as a replacement to NumPy which enables the transfer of the most computationally expensive portions of the code to the GPU. This speeds up the simulations by a factor of up to 30 for our typical test problems on a modern and widely accessible GPU, allowing for larger system sizes while still retaining the advantage of having a fast and easily accessible development cycle.

The computational bottleneck of a center-based model implementation is the calculation of the total force acting on each cell. According to this force the cell position is updated in every time step in Eq. (4). In order to calculate the total force vector for the complete cell system, all pairwise forces need to be evaluated. In other major centerbased software, computing the force interactions between cell pairs is usually done using a bounding box technique, where space is discretized into voxels larger than the maximum interacting distance between cells [42]. The forces applied to a given cell are then only computed for the cells located in the same voxel and in neighboring voxels. Given that the system usually relaxes to a given density of cells per voxel, i.e. the number of cells per voxel will be bounded from above, this algorithm achieves linear complexity with respect to the number of cells. Unfortunately, implementing such an algorithm in pure Python code is typically orders of magnitude slower than in compiled languages.

Calculating the interactions between all possible cell pairs, on the other hand, can be -contrary to the bounding box algorithm- easily expressed with array programming instead, a programming paradigm based on elementary array operations, such as indexing, vectorization, broadcasting and reduction. In Python, NumPy is now the de facto standard for array programming [43]. By using an optimized, pre-compiled layer of $\mathrm{C}$ code under the hood, NumPy provides improved performance for all array operations, while still making it possible to write legible Python programs. This means that while this naive implementation scales quadratically with the number of cells, it still achieves decent performance thanks to NumPy. On top of that, there exist a myriad of Python modules implementing NumPy's array protocol. Such libraries include Dask [44], for distributed large arrays or PyData/Sparse [45] for sparse matrices. For GPU computations in particular, there exist several high-level libraries aiming to extend NumPy, such as CuPy [29], MinPy [46] (deprecated, now merged with MXNet Gluon [47]) and afNumPy [48]. In practice, such array implementations provide drop-in replacements for a large subset of NumPy functions and thus require only minimal modifications of the code to be used.

If a GPU is available, a CBMOS user can specify the use of the CuPy library as a high performance computing backend. Although originally developed specifically for 3D graphics, GPUs have become widely available for general computations in recent years with the advent of the CUDA programming language and GPGPUs (general-purpose computation on GPUs) [49]. When threads are relatively independent and only have 
to synchronize for atomic operations, GPUs make it possible to run massively parallel applications executing thousands of threads at a time. In the case of CBMOS, the bulk of the computations is done when computing the total force which can be expressed as independent, predictable operations applying to all elements of an array, making it wellsuited for GPU computations. The arrays involved in the computation of the total force are created on the GPU and calculation of the force vector is done there. This GPU-enabled version reduces the computation time for a single evaluation of the force vector for 10,000 cells to $0.4 \mathrm{~s}$, which is about thirty times faster than on the CPU.

\section{Code availability and usage}

The full program is readily available under the MIT license on the Python package index [50] and on GitHub [30]. Installing and running CBMOS is straightforward as it only depends on a few well maintained external modules (mainly the Python scientific software stack NumPy and SciPy, and optionally CuPy). The documentation is available on the project's GitHub page [31] and describes how to set up a simple simulation. An example of a convergence study is also presented there.

Users interested in extending CBMOS' functionality will find it easy to add force functions, solvers or even utility functions for e.g. generating and plotting population configurations to the appropriate submodules.

One of the main advantages of CBMOS over other similar software is the possibility to run simulations, analyse them and interpret the results all in a single Jupyter Notebook. Jupyter Notebooks have become very popular in recent years and are an excellent way to report reproducible scientific findings [41]. In fact, the recent development of online platforms providing free, ready-to-use resources to execute such notebooks (even on GPUs) makes this process even easier. For instance, all the notebooks used in this study are freely available on our GitHub repository and can be set up and rerun in a couple minutes on Google Colab [32].

\section{Results and discussion}

We now proceed to demonstrate how CBMOS can be used to perform numerical experiments. The following section has two main parts. First, we focus on the computational performance of CBMOS both on CPU and GPU and draw practical bounds as to which one is most suitable, depending on the number of cells considered in the simulation. Second, to show-case what kind of questions can be addressed using CBMOS, we conduct a numerical study comparing the implicit backward Euler method for solving the update equation to the more commonly used explicit forward Euler method. Note that while all numerical experiments were done in two dimensions, CBMOS is capable of simulating three dimensional cell populations as well.

\section{Performance comparison CPU/GPU}

In this section, we illustrate the performance gain enabled by evaluating the total force vector on the GPU instead of the CPU, pushing the limit of how many cells a centerbased code written in Python can simulate. First, we ran a performance benchmark to study wall time as a function of the number of cells for a compressed monolayer relaxing 
to steady state. Second, we prescribed a fixed wall time and counted how many cells could be simulated in a monolayer growth experiment within that time. For both experiments we compared our array-programming-based implementation to the bounding box algorithm described in the implementation section. Taken together, these experiments provide a practical estimate for the most suitable algorithm in terms of cell population sizes.

The benchmarks were run on Snowy, an HPC cluster provided by the Multidisciplinary Center for Advanced Computational Science (UPPMAX). The node we used consisted of two 8-core Xeon E5-2660 processors at 2.2 GHz, $128 \mathrm{~GB}$ of memory, and was equipped with an Nvidia T4 GPU.

\section{Relaxation benchmark}

In the first benchmark scenario we generated cell populations of different sizes arranged in a compressed honeycomb pattern in which the distance between any two neighboring cells was initialized to 0.8 cell diameters (the rest length was set to $s=1.0$ cell diameter). We then allowed the system to relax to steady state over the course of one in-simulation hour. Within this time no proliferation took place, so that no cellular events needed to be handled. The force function was chosen as the generalized linear spring (GLS) force with parameter settings $\mu=1.95$ and $a=-2 \log (0.002 / \mu)$. The choice of these parameters resulted in a relaxation time between daughter cells after cell division of $1 \mathrm{~h}$ (insimulation time), as described in [27]. The time step was set to $\Delta t=0.1 \mathrm{~h}$, ensuring that cell trajectories after cell division remain physically correct (again, for details we refer to our previous numerical study [27]).

Figure 2a shows the total execution time for the relaxation experiment with the three implementations described in the implementation section: (1) the bounding box

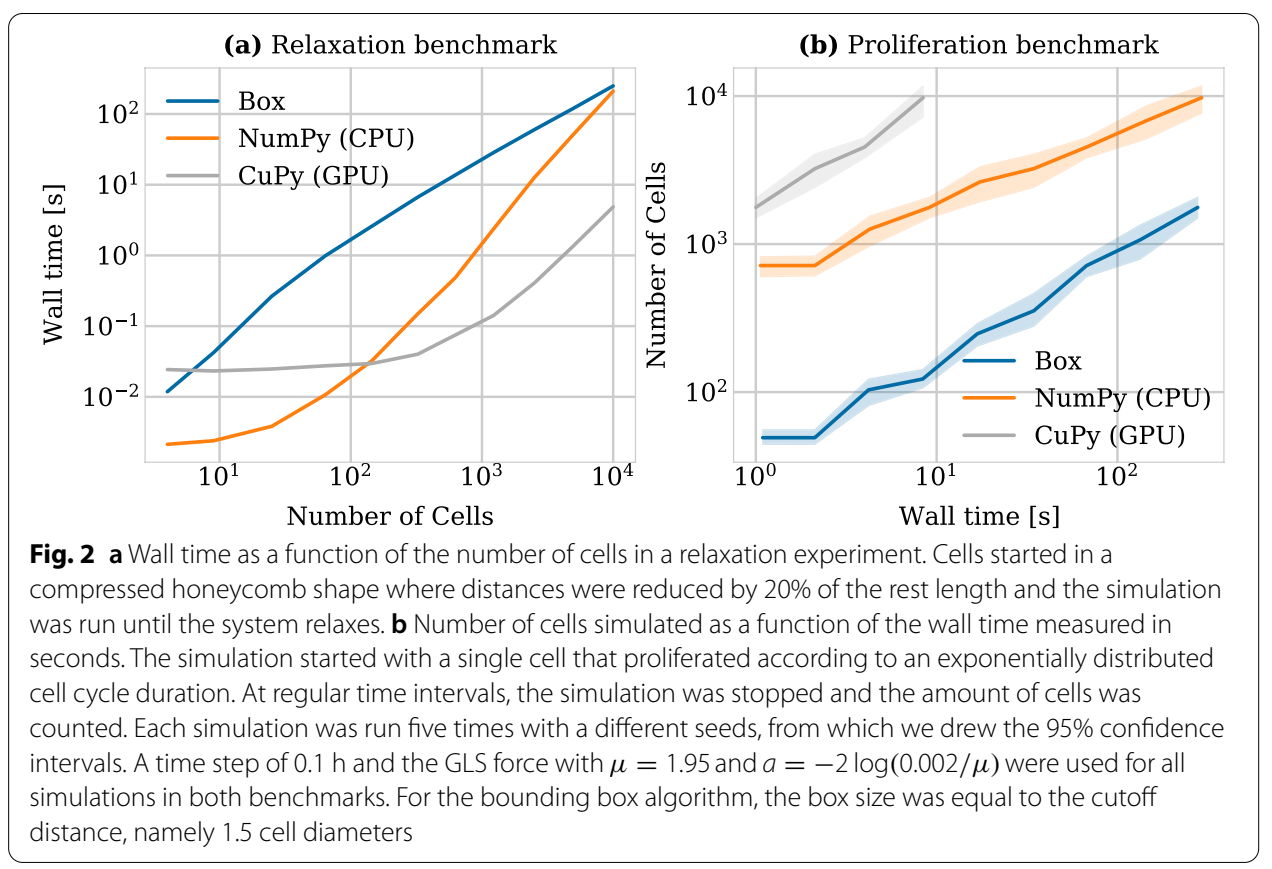


implementation (denoted by 'Box' in the legend), (2) the array implementation using NumPy and running only on the CPU (denoted by 'NumPy') and (3) the array implementation using $\mathrm{CuPy}$ in addition to NumPy (denoted by 'CuPy'), which transfers the calculation of the force vector at each time step to the GPU. Although the bounding box implementation has the best computational complexity, it was an order of magnitude slower than both array implementations for all practical use cases considered here (as a reference, in the previous CBMOS publication [27], we only considered experiments with 2, 38, 74 and 400 cells). The bounding box implementation only beats the NumPy implementation starting at $10^{4}$ cells and above, at which point the simulation took around five minutes to complete.

Transferring the calculation of the force vector-the main computational bottleneckto the GPU brought significant improvements starting already from 100 cells. More specifically, the GPU-enhanced array implementation was up to about one and a half orders of magnitude faster for the range of 100-10,000 cells. For lower numbers of cells its performance was dominated by the overhead of transferring the data to and from the GPU. Most notably, for 10,000 cells, the simulation only took $4 \mathrm{~s}$, compared to around five minutes for the bounding box implementation and the CPU-only array implementation. Above this point, the GPU we used ran out of memory. In terms of pure execution times (given enough memory), however, we can theoretically estimate this implementation to be faster than the bounding box implementation for up to $6.25 \times 10^{5}$ cells by extrapolating from the algorithm's quadratic complexity.

Overall, this benchmark illustrates the range of application of the CBMOS package in terms of cell population sizes for non-proliferating two dimensional populations and in the context of exploratory prototyping, where single realizations should run within a minute. For up to a 100 cells, the NumPy implementation is sufficient. Above this threshold, the use of a GPU enables significant performance gains for the simulation of up to 10,000 cells, decreasing the simulation time from several minutes to just a few seconds. For larger system sizes, the memory requirements become prohibitive and other center-based software frameworks should be considered.

\section{Proliferation benchmark}

In a second benchmark, we studied the question of how many cells one could simulate with the different versions within a fixed execution time in a monolayer growth experiment. To this end, we set up a single initial cell. This initial ancestor proliferated according to an exponentially distributed cell cycle duration (with a mean of $1.0 \mathrm{~h}$ ), generating a large cell population over time. We stopped the simulation after a fixed wall time had elapsed and counted the number of the cells in the population. We considered simulation times of up to a few $100 \mathrm{~s}$, which in our opinion is about the maximum reasonable waiting time one can afford to wait when prototyping. Division events were only allowed to take place every $0.1 \mathrm{~h}$. All other parameters, in terms of force functions and time steps, were the same as in the relaxation benchmark.

Figure $2 \mathrm{~b}$ shows our results by plotting the number of cells simulated as a function of wall time. Again, in spite of its quadratic complexity, the NumPy implementation outperforms the bounding box implementation by an order of magnitude in terms of the number of simulated cells for all practical simulation times. Deploying the code on a 
GPU showed even greater performance, simulating around 10,000 cells in about $8 \mathrm{~s}$, at which point the GPU ran out of memory.

In the previous relaxation benchmark the number of cells was fixed from the beginning and no proliferation took place, meaning no cell events needed to be handled during the simulation. This allowed the solver to run continuously from the beginning to the end. In this benchmark, however, proliferation was included and division events took place throughout the simulation, usually forcing the solver to restart after every time step once the cell population had grown past a certain size (due to the large number of cells and consequently division events). This benchmark shows that even in this case the CuPy implementation largely outperforms the two other options.

Having shown that our implementation is reasonably efficient for system sizes of up to 10,000 cells (when using the GPU) in different experimental settings, we now move on to study the use of an implicit method for solving the system of ODEs for the cell midpoints.

\section{Numerical study of the implicit backward Euler method}

In this section we show-case the ability of CBMOS to study the numerical properties of center-based models, by illustrating two common workflows: (1) a comparison of the numerical properties of two solvers by using CBMOS within a Jupyter notebook and (2) measuring the average wall time of both solvers when used to calculate cell trajectories of two dimensional cell populations, where the latter experiment is run on a high performance computing cluster. In particular, we investigate whether the use of an implicit method is beneficial in terms of computational cost necessary to achieve a desired accuracy. Before describing the numerical experiments we conduct, we give a brief overview of the two numerical methods in question, the forward and backward Euler methods.

\section{Mathematical definition of the forward and backward Euler methods}

A commonly used numerical method for solving the ODE system for the center positions in center-based model implementations is the forward Euler method [21]. It is an explicit method, meaning the function value at the next time point can be explicitly calculated from the current function value. For an initial value problem stated as

$$
\dot{\mathbf{x}}=\mathbf{F}(\mathbf{x}), \mathbf{x}\left(t_{0}\right)=\mathbf{x}_{0}
$$

the forward Euler method can be written as

$$
\mathbf{x}_{n+1}^{\mathrm{EF}}=\mathbf{x}_{n}+\Delta t \mathbf{F}\left(\mathbf{x}_{n}\right),
$$

where $\mathbf{x}_{n}$ denotes the current function value, $\mathbf{x}_{n+1}$ the function value at the next time point $t_{n+1}=t_{n}+\Delta t$ and $\Delta t$ is the step size. As an explicit method, the forward Euler method suffers from stability issues for stiff systems. More specifically, if the time step size $\Delta t$ is chosen too large, the numerical solution will oscillate and grow without bounds, even though the true solution is smooth and decreases. Equation systems with steep force gradients - as is the case in Eq. (4) after cell proliferation-can be expected to behave stiffly. The backward Euler method-the simplest implicit method for solving 
ODE systems-does not exhibit this constraint on the time step size and is a common solution to stiffness issues [51]. It can be written as

$$
\mathbf{x}_{n+1}^{\mathrm{EB}}=\mathbf{x}_{n}+\Delta t \mathbf{F}\left(\mathbf{x}_{n+1}\right) .
$$

Note that here, in contrast to Eq. (6), the total force $\mathbf{F}$ (which in general is non-linear) is evaluated at the next time point. Thus, obtaining a value for the next function value $\mathbf{x}_{n+1}$ with the backward Euler method requires the iterative solving of Eq. (7) with a linear system solve in each step.

Implicit methods such as the backward Euler method gain their improved stability properties at an increased computational cost. As they formulate the equation for the next function value depending on the gradient at the next time point (instead of the current as explicit methods do), they require this equation to be solved iteratively with a linear system solve in each step. The function value $\mathbf{x}_{n+1}$ in Eq. (7) is calculated by solving

$$
G\left(\mathbf{x}_{n+1}\right)=0
$$

by Newton iterations, where $G(\mathbf{x})=\mathbf{x}-\mathbf{x}_{n}-\Delta t \mathbf{F}(\mathbf{x})$. More specifically, $\mathbf{x}_{n+1}^{(0)}$ is initialized using $\mathbf{x}_{n}$ and then for several iterations $j$ the following two steps are executed,

$$
\begin{aligned}
& \text { 1.J } \mathbf{J} \mathbf{x}=-G\left(\mathbf{x}_{n+1}^{(j)}\right) \\
& 2 . \mathbf{x}_{n+1}^{(j+1)}=\mathbf{x}_{n+1}^{(j)}+\Delta \mathbf{x} .
\end{aligned}
$$

In the first step $\mathbf{J}=\mathbf{J}\left(\mathbf{x}_{n+1}^{(j)}\right)$ denotes the Jacobian of $\mathrm{G}$ which directly depends on the Jacobian of the ODE system $\mathbf{A}=\left\{\partial \mathbf{F}_{k} / \partial \mathbf{x}_{l}\right\}_{k, l}$ as $\mathbf{J}=\mathbf{I}-\Delta t \mathbf{A}$, where $\mathbf{I}$ is the identity matrix. J defines the linear system that needs to be solved for $\Delta \mathbf{x}$. This can be done e.g. with the generalized minimal residual method (GMRES) [52].

CBMOS provides an analytically correct implementation of the Jacobian J. In practice, however, it is more efficient to approximate the matrix-vector product Jv in GMRES via

$$
\mathbf{J v}=\frac{1}{\xi}\left(G\left(\mathbf{x}_{n+1}^{(j)}+\xi \mathbf{v}\right)-G\left(\mathbf{x}_{n+1}^{(j)}\right)\right)
$$

without having to assemble the Jacobian $\mathbf{J}$ [53]. Here, $\xi$ denotes an approximation parameter, for which we use $\xi=0.001$ in all our numerical experiments.

In general, the improved stability properties of the backward Euler method should allow for larger time step sizes necessary to achieve a given accuracy, at least for moderate accuracy requirements. However, it is not clear in practice whether the increase in time step size can compensate for its additional cost or if using the forward Euler method with more-yet cheaper-small time steps is more efficient. In the next section, we use the investigation of this question to illustrate CBMOS' potential.

\section{Numerical comparison of the forward and backward Euler methods}

First, we demonstrate how CBMOS can be used in a regular Jupyter Notebook to conduct simple numerical studies. The notebook used for this case study is provided along this manuscript as Additional file 1. More specifically, we examine the different stability properties of the forward and backward Euler methods using the simple test case of two cells relaxing after cell division. The cells were initially placed at an overlap of 0.3 cell 


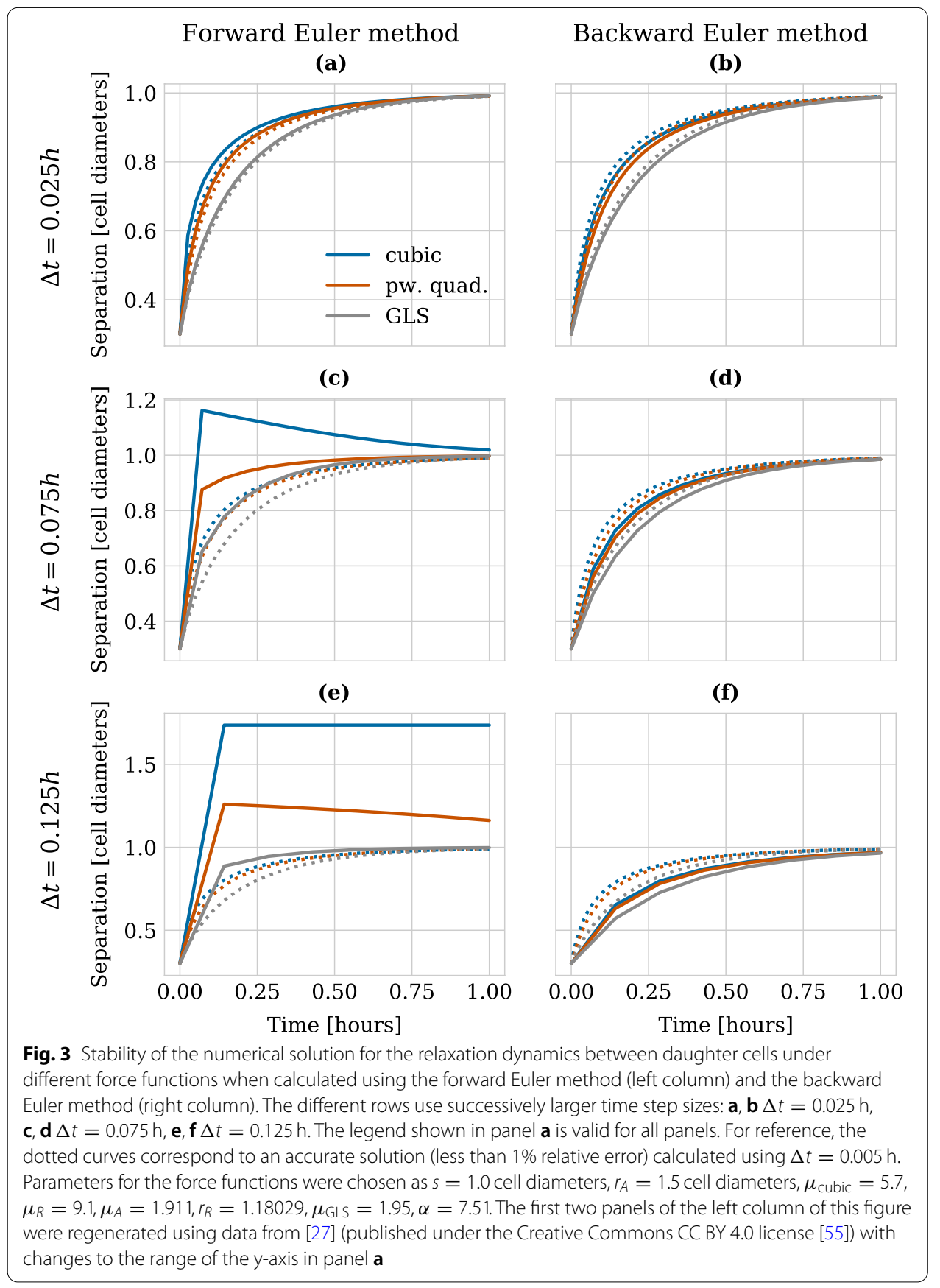

diameters which they eliminated until they were at rest at a distance of one cell diameter with no forces acting between them. Parameters have been chosen such that the duration of this process-called the relaxation time-was (arbitrarily) fixed as $1 \mathrm{~h}$. As shown in our previous study [27], using the forward Euler method requires the time step size to be chosen lower than a certain stability threshold to recover a numerically stable solution. Moreover, if cell trajectories after division should be physically correct, i.e. cells should not jump apart before adhering again, then the time step size is even more 
restricted by a monotonicity bound which is half the stability bound for this specific test case. The exact values of these bounds are force function and parameter dependent (see [27] for details). Varying the time step size, numerical solver and force function can easily be done in CBMOS by passing these as arguments when creating the model or launching the simulation.

Figure 3 shows the behavior of the numerical solution for the cell trajectories after cell division when calculated using the forward (left column) and backward (right column) Euler methods. Subsequent rows differ in the size of the time step used, ranging from a very small time step size of $\Delta t=0.025 \mathrm{~h}$ in panels (a, b), to a large step size of $\Delta t=0.125 \mathrm{~h}$ in panels $(\mathrm{e}, \mathrm{f})$. All panels show the trajectories for three different pairwise force function choices, illustrating that stability is both a property of the solver and the ODE system itself (defined via the pairwise force function). For reference, the dotted curves in each panel correspond to an accurate solution (less than $1 \%$ relative error) calculated using $\Delta t=0.005 \mathrm{~h}$. In the left column, where the explicit forward Euler method was used, we observe that for the smallest time step size all trajectories are physically correct, whereas for the larger step sizes the trajectories show physically correct (piecewise polynomial force in (c) and GLS force in (c, e)), physically incorrect yet stable (cubic force in (c) and piecewise polynomial force in (e)) or even numerically unstable behavior (cubic force in (e)), depending on how sensitive the force functions are to the time step choice (note that the first two panels from the left column were regenerated using the same data as in our previous publication [27]).

In contrast, the right column of Fig. 3 shows the exact same experimental setup for the implicit backward Euler method. Here, we observe that independently of the time step size and the force function choice the trajectories remain physically correct. (Note of course that for larger time step sizes the accuracy decreases as expected). Our further investigations in [27] illustrated that a choice of a too large time step in combination with the forward Euler method-in particular a time step size violating the monotonicity bound at the pairwise cell level-could result in geometrical differences even at the population level. It is therefore of interest to resolve the pairwise dynamics correctly and hence the question arises whether the backward Euler method is a more suitable choice because it allows for larger time step sizes without stability nor monotonicity constraints.

We now turn to another common setup when doing numerical studies, where the duration of the computations makes it impractical to run the numerical experiment itself within a Jupyter notebook. This case arises when the time step size required for the experiment is very small, when the simulation includes many cells, or when many repeated simulations are necessary. In this setup, the experiment is typically run on a high performance computing cluster using batch scripts and the results are processed in a Jupyter notebook afterwards. Specifically, we were interested in evaluating the cost of using the backward Euler method versus the forward Euler method to investigate whether using the backward Euler method could be more efficient for moderate accuracy values of 5-10\%. Once again, CBMOS' application programming interface (API) makes it straightforward to switch between numerical configurations and to compare the results. 


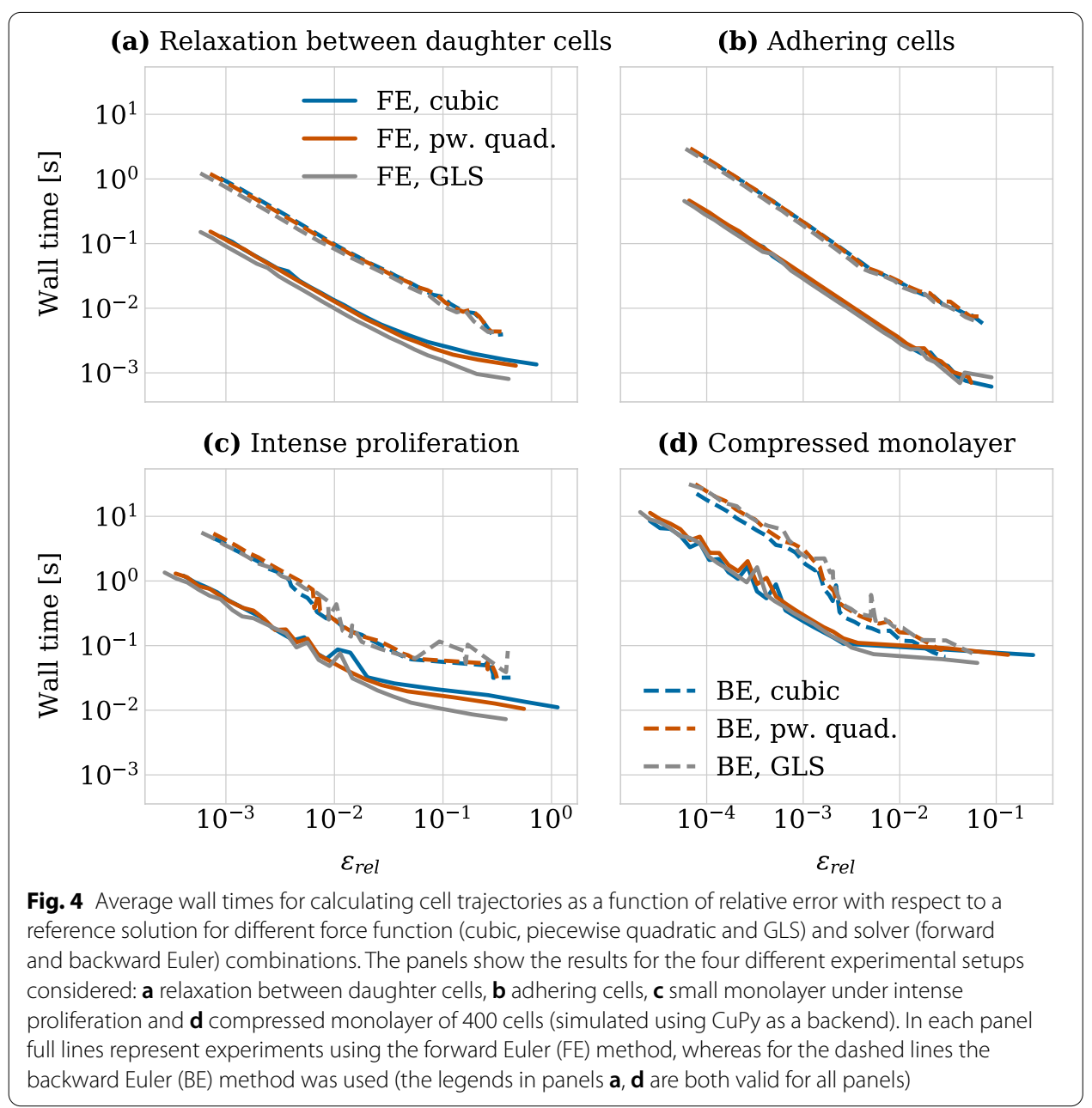

Table 1 Table showing the relative error values $\varepsilon_{\text {rel }}$ and the time step values $\Delta t$ (in hours) for the forward and backward Euler methods in combination with different force functions

\begin{tabular}{llllll}
\hline & \multicolumn{2}{l}{ Forward Euler } & & \multicolumn{2}{l}{ Backward Euler } \\
\cline { 2 - 3 } & $\boldsymbol{\varepsilon}_{\boldsymbol{r e l}}$ & $\boldsymbol{\Delta}$ & & $\boldsymbol{\varepsilon}_{\text {rel }}$ & $\boldsymbol{\Delta} \boldsymbol{t}$ \\
\hline Cubic force & 0.031 & $0.108 \mathrm{~h}$ & & 0.030 & $0.808 \mathrm{~h}$ \\
Pw. quad. force & 0.031 & $0.136 \mathrm{~h}$ & & 0.029 & $0.808 \mathrm{~h}$ \\
GLS force & 0.027 & $0.212 \mathrm{~h}$ & & 0.030 & $0.646 \mathrm{~h}$
\end{tabular}

The experimental setup chosen was the relaxation of a compressed monolayer of 400 cells. Values were rounded to three decimals

To do so, we averaged wall times over 10 repetitions of calculating the cell trajectories for the convergence study presented in the appendix (c.f. Fig. 5). Figure 4 shows this average as a function of the relative error for four different experimental setups: (1) relaxation between two daughter cells after cell division-same as before when comparing the stability properties in the results section-; (2) adhering cells placed at an initial distance of 1.15 cell diameters; (3) a small monolayer population of initially 19 cells, all cells were allowed to divide at the beginning of the simulation, leading to a highly compressed cell population which then relaxed to a steady state configuration 
over the course of two in-simulation hours; (4) relaxation of a large monolayer, similarly to the setup of the relaxation benchmark. Here, we initialized a monolayer population of 400 cells for which we were able to conduct the convergence study in a reasonable time by using CuPy as a backend. We note that for moderate accuracy requirements, the backward Euler method is able to use larger time step sizes than the forward Euler method. Nevertheless, using the backward Euler method is about one order of magnitude slower than using the forward Euler method across the complete range of relative error values for both experimental setups with two cells and the intense proliferation test case. It is only for the case of the large compressed monolayer and a relative error of roughly $3 \%$ or larger that the backward Euler method achieves a similar wall time to the forward Euler method. At this point the backward Euler method was able to use time step sizes up to nearly 8 times as large depending on the force function chosen. The exact values for relative errors and time step sizes are compared in Table 1.

Implicit methods, such as the backward Euler method, can be beneficial when stability, rather than accuracy, is the limiting factor [21]. In this subsection, we have investigated if this was indeed the case for ODE systems from center-based models. We conclude that based on our numerical experiments it is not beneficial to use the backward Euler method compared to the forward Euler method, even if it allows for larger time step sizes for low accuracy values. Its additional computational cost due to requiring a linear solve remains too high even when approximating the Jacobian and using as few iterations as possible.

Most importantly, these numerical experiments could not have been performed with the same ease with other existing center-based simulation software. Indeed, the Python programming language makes it simple to iterate over the model parameter combinations and repeat the same experiment while keeping all the other parameters constant. Moreover, because these parameters are exposed to the user by CBMOS' API, there is no requirement to dive into CBMOS' source code to change the numerical behavior of the simulation. All these factors make CBMOS a suitable tool for prototyping experiments, where one needs to compare similar setups and fine tune numerical parameters. Furthermore, the ability to write such experiment in Jupyter notebooks makes it possible to present the results and the code in a concise, reproducible report [54]. As already stated previously, the notebook used for the stability experiments is provided along this manuscript as Additional file 1. All other notebooks and data necessary to reproduce the results presented in this article can be found in the CBMOS GitHub repository.

\section{Conclusions}

In this article we presented CBMOS, an open-source package for the numerical study of center-based models. CBMOS provides a flexible interface to study the effect of core components of the numerical simulation of center-based models-amongst others the force function used for the pairwise interaction forces between cells and the numerical method for solving the update equation for the cell midpoint coordinates-on the mechanics of a proliferating cell population as well as on the efficiency of the simulation itself. To this end, it includes implementations of many popular force functions as well 
as several explicit and implicit first- and second-order numerical methods. Its interface is designed to allow for easy extension in terms of more force functions or numerical methods, but also more types of cellular events.

Written in Python, CBMOS provides easy access for modelers of any level and enables fast prototyping workflows. Thanks to relying on NumPy's array programming paradigm it is reasonably efficient simulating small cell populations on the order of hundreds of cells on the CPU. Moreover, it extends this range to cell populations of up to 10,000 cells in connection with a high-end GPU through the CuPy library. In that case execution times for simulating monolayer growth are on the order of a few seconds, with the amount of memory available on the specific GPU becoming the limiting factor. In its current state on our test hardware CBMOS was capable of running repeated convergence studies on test systems with thousands of cells within a few hours.

Naturally, an implementation in a compiled language (as used for other open source simulation packages for center-based models such as e.g. PhysiCell [16] or Chaste [14]) or accessing CUDA features more directly (as done e.g. in ya||a [17]) can be expected to be more performant in terms of pure execution speed. However, those kinds of implementations usually require a substantial investment in terms of both development and implementation time, not to mention that they may require a steep learning curve for users less familiar with programming. It is our hope that developing a Python package will have the additional benefit of being more easily accessible for modelers not coming from a computational background. In general, the combination of a interpreted language with high-level GPU-libraries such as CuPy can be very attractive and advantageous as the former permits fast-prototyping workflows, while the latter ensures significant performance gains at virtually no added implementation time cost.

CBMOS is implemented in an event-driven fashion, meaning it simulates the mechanics of the cell population until the next cellular event happens. This has the advantage that cellular events are applied at the exact time they occur. For large populations with many cellular events this approach can become inefficient due to the need to simulate the mechanics for fractions of a time step in order to advance the system to the correct state before applying the cellular event. Therefore, we have chosen to augment CBMOS with the option to aggregate cellular events to a fixed time resolution. This means that at pre-determined time points we apply all cellular events that would have taken place between the last check point and the current time. This time-driven approach is how most center-based model frameworks are implemented. It results in a lower bound to the step size of the mechanics simulation at the cost of an additional splitting error. Thanks to implementing both approaches CBMOS could be used in future work to investigate the error of the time-driven versus the event-driven approach. This would allow to formulate guidelines on how to choose the fixed time resolution of the cellular events in order to balance incurred error with simulation efficiency.

In a previous publication [27] we used a prototype of CBMOS to investigate how popular force functions should be parametrized to reproduce consistent mechanical behavior for two dimensional cell populations, as well as how the combination of force function and numerical solver affects the efficiency of center-based models in terms of time step sizes. For the latter we focused on explicit first and second-order solvers. In this article, to further illustrate the types of questions CBMOS can be used to address, we studied the 
complementary question of whether an implicit method for solving the update equations can be beneficial. More specifically, we studied whether the better stability properties of the backward Euler method can balance out its increased computational cost compared to the forward Euler method for different experimental setups. In line with our previous study we considered three popular force function choices on the test cases of (1) daughter cells relaxing after cell division, (2) adhering cells and (3) a small monolayer population undergoing intense proliferation. Furthermore, we considered a large compressed monolayer of 400 cells as an additional setup, the simulation of which was possible thanks to using $\mathrm{CuPy}$ as a GPU-backend. For all experimental setups the backward Euler method was less efficient in terms of wall time for computing the cell trajectories than the forward Euler method, although it was able to use larger time step sizes for moderate to small accuracy values. On average, the backward Euler method was around one order of magnitude slower than the forward Euler method, even with generous threshold settings and when approximating the Jacobian at the cost of an extra evaluation of the total force (instead of assembling the complete analytical Jacobian at a higher cost). These results extend $\mathrm{K}$. Atwell's findings in her thesis [26], where she investigated the backward Euler method as well as another second-order implicit method for a small tumor-growth experiment.

To summarize, our results confirm that using the forward Euler method with sufficiently small time step sizes is computationally more efficient than using the backward Euler method, at least as long as fixed time-stepping is used. With adaptive time stepping, where the time step size is chosen dynamically according to a suitable error estimate, there might be gains with the backward Euler method for systems that spend long durations in states where overall the forces between cells are weak, e.g. when strong compression forces due to cell division events are rare. Additionally, in such a setting it might be even more advantageous to switch between both methods, depending on whether the time step size is restricted by accuracy or stability concerns. We leave the exploration of both ideas for future work.

There exist several other center-based model frameworks implementing a larger feature set and better performance due to being written in a compiled language. Nevertheless, we believe that, by making it possible to isolate fundamental aspects of the simulation through a user-friendly API, CBMOS offers easy access to any modeler wishing to quickly challenge key numerical properties of their center-based model or to test preliminary hypotheses before committing to a more complex simulation framework.

\section{Appendix}

\section{Convergence study}

Aside from stability, an important property of a numerical method is its order of convergence, describing how fast its accuracy increases when decreasing the time step size. It can be shown theoretically that both the forward and backward Euler methods are first-order methods, meaning that roughly speaking halving the step size also halves the error in the numerical solution. To confirm this numerically, we conducted a convergence study where we analysed the error as a function of the time step size for both methods and different example systems. We generated cell trajectories for multiple time 


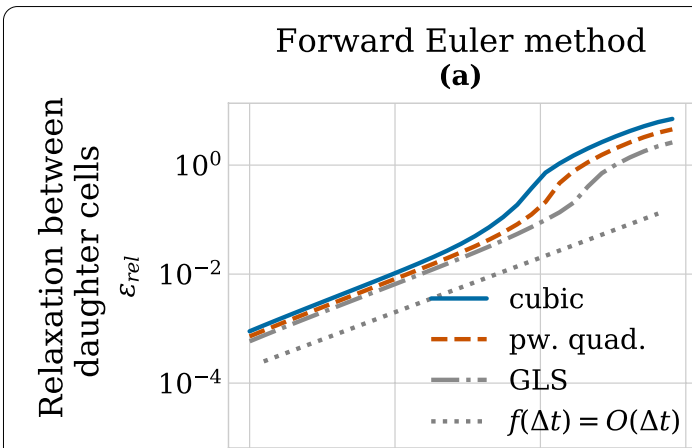

(c)

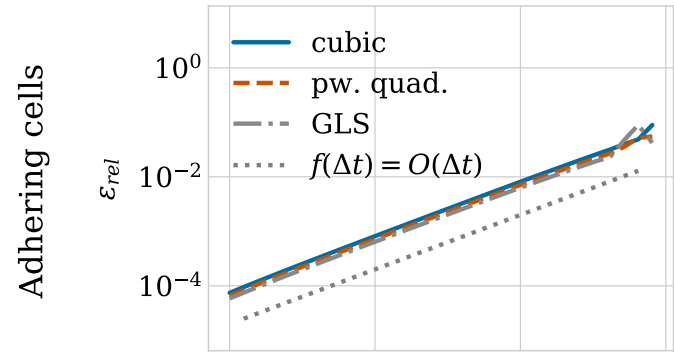

(e)

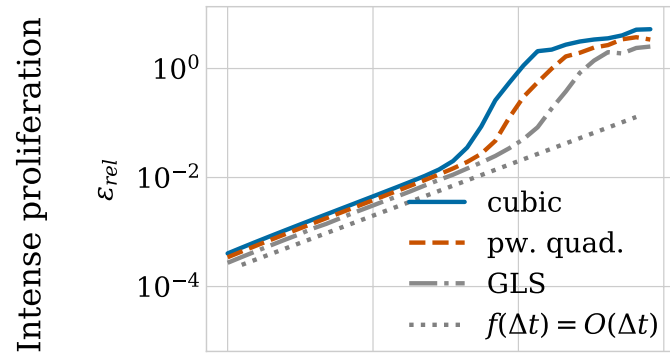

(g)

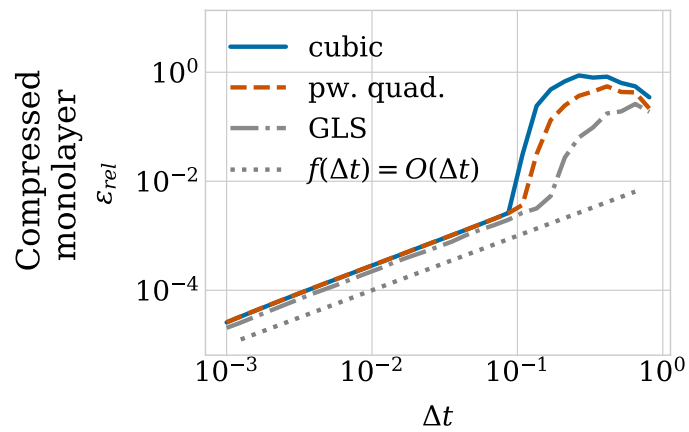

Backward Euler method

(b)

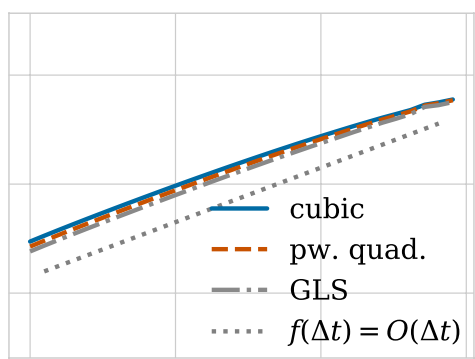

(d)

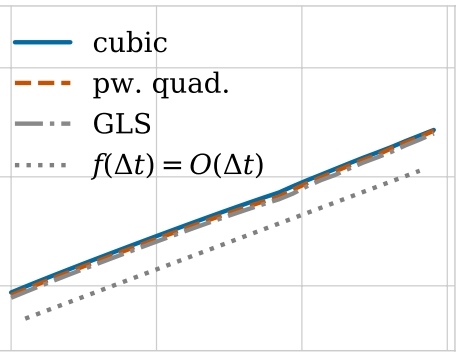

(f)

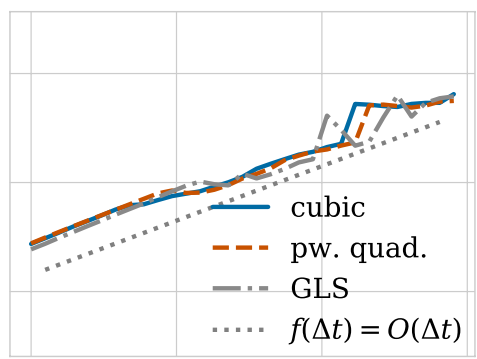

(h)

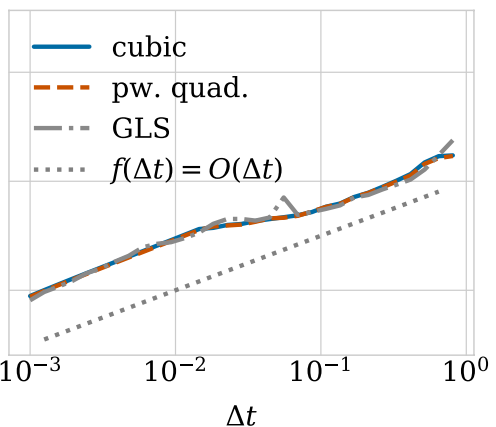

Fig. 5 Impact of time step size on the relative error for the different combinations of forces and both numerical solvers tested on four model problems (1)-(4). $\mathbf{a}, \mathbf{b}$ Convergence study for the relaxation experiment (1), initial separation 0.3 cell diameters, $\mathbf{c}, \mathbf{d}$ convergence study for adhering cells (2), initial separation 1.15 cell diameters, $\mathbf{e}, \mathbf{f}$ convergence study for a monolayer population of initially 19 cells under intense proliferation (3) and $\mathbf{g}, \mathbf{h}$ convergence study for a compressed monolayer population of 400 cells (4). The numerical solver used was in $\mathbf{a}, \mathbf{c}, \mathbf{e}, \mathbf{g}$ the forward Euler method, and in $\mathbf{b}, \mathbf{d}, \mathbf{f}, \mathbf{h}$ the backward Euler method. The axes of the plots are in logarithmic scale. The dotted lines show a linear function to facilitate observation of the convergence order. Note that the first three panels in the left column include data used also in our previous publication [27] (published under the Creative Commons CC BY 4.0 license [55]). Compared to the original work, the ranges of the $x$ and $y$-axes as well as the legends have been modified and additional data has been included 
step values and calculated the error for each value $\Delta t$ relatively to a reference solution $\mathbf{x}_{\text {ref }}$ generated with a very small time step of $\Delta t_{\text {ref }}=10^{-4} h$ as

$$
\varepsilon_{\text {rel }}=\frac{\left\|\mathbf{x}_{\Delta t}-\mathbf{x}_{\mathrm{ref}}\right\|_{2}}{\left\|\mathbf{x}_{\mathrm{ref}}\right\|_{2}}
$$

where the coarser solution $\mathbf{x}_{\Delta t}$ was interpolated down to the fine time grid used for the generation of $\mathbf{x}_{\text {ref }}$. Moreover, we measured the wall time for the generation of the cell trajectories (averaged over 10 repetitions), these data form the basis for Fig. 4 in the main text.

Figure 5 shows the results of our convergence study for four different experimental setups. The first setup, shown in panels $(\mathrm{a}, \mathrm{b})$, considered the relaxation between two daughter cells after cell division, same as before when comparing the stability properties in the results section. In the second, shown in panels (c, d), we placed two cells at an initial distance of 1.15 cell diameters and let them adhere until they touched. The third setup in panels (e, f) was chosen as a small monolayer population of initially 19 cells. All cells were allowed to divide at the beginning of the simulation, leading to a highly compressed cell population which then relaxed to a steady state configuration over the course of two in-simulation hours. Finally, as a fourth experimental setup we considered the relaxation of a large monolayer, similarly to the setup of the relaxation benchmark. Here, we initialized a monolayer population of 400 cells for which we were able to conduct the convergence study in a reasonable time by using CuPy as a backend. The results for this experimental setup are shown in panels $(g, h)$.

In the left column of Fig. 5 the forward Euler method was used to calculate cell trajectories, whereas the right column shows the results for the backward Euler method. All plots show the relative error as a function of time step size for three different force functions with the axes in logarithmic scale. We observe that both numerical methods show the correct first-order convergence across all force functions and experimental setups for small time step sizes $\Delta t<10^{-2} \mathrm{~h}$. For larger time steps in the range of $(0.05 \mathrm{~h})$ to $(0.5 \mathrm{~h})$ the forward Euler method shows a larger relative error for all experimental setups except the second one with the adhering cells. This is due to the loss of monotonicity and stability in the cell trajectories for these time step sizes, as discussed in the previous section. This means that the backward Euler method is able to achieve relative error values of the order of $10^{-1}$ with larger time step sizes than the forward Euler method. This raises the question whether the backward Euler method can be beneficial if cell trajectories need to be resolved with only moderate accuracy, i.e. when a relative error of (5-10\%) is acceptable.

\section{Abbreviations}

API: Application Programming Interface; CPU: Central processing unit; GPU: Graphics processing unit; GLS: Generalized linear spring; ODE: Ordinary differential equation; FE: Forward Euler method; BE: Backward Euler method.

\section{Supplementary Information}

The online version contains supplementary material available at https://doi.org/10.1186/s12859-022-04575-4.

Additional file 1. Stability Study of FE and BE. Jupyter notebook used to perform the stability study described in the Numerical comparison of the forward and backward Euler methods section. 


\section{Acknowledgements}

We would like to thank Per Lötstedt for fruitful discussions about the implicit Euler method, its implementation and how to measure its cost. We would also like to thank Carl Nettelblad for providing a singularity container with CuPy installed. All numerical experiments were performed on the Snowy compute resources provided through the Uppsala Multidisciplinary Centre for Advanced Computational Science (UPPMAX) within the Project SNIC 2019-8-227.

\section{Authors contributions}

SM and AC developed and maintained the CBMOS software, ran the computational experiments and prepared the initial draft of the manuscript. The ideas were developed in close collaboration with AH. All authors reviewed and edited the draft. All authors read and approved the final manuscript.

\section{Funding}

Open access funding provided by Uppsala University. This work has received funding from the Swedish Research Council under grant 2015-03964, from the NIH under grant no. NIH/2R01EB014877-04A1 and from the eSSENCE strategic initiatives on eScience. The funders had no role in the design of the study, data collection, data analysis, interpretation of results, or writing of the manuscript.

\section{Availability of data and materials}

CBMOS can be accessed from https://github.com/somathias/cbmos or installed via PyPI. The results and figures presented in this article can be directly reproduced from the Jupyter notebooks available in the GitHub repository. Availability and requirements: Project name: CBMOS. Project home page: https://github.com/somathias/cbmos. Operating system(s): Platform independent. Programming language: Python 3. Other requirements: Numpy $\geq 1.18$, SciPy $\geq$ 1.4.0, CuPy (optional). License: MIT. Any restrictions to use by non-academics: MIT licensing restrictions apply.

\section{Declaration}

Ethics approval and consent to participate

Not applicable.

\section{Consent for publication}

Not applicable.

\section{Competing interests}

The authors declare that they have no competing interests.

Received: 26 August 2021 Accepted: 11 January 2022

Published online: 31 January 2022

\section{References}

1. Van Liedekerke P, Palm M, Jagiella N, Drasdo D. Simulating tissue mechanics with agent-based models: concepts, perspectives and some novel results. Comput Particle Mech. 2015;2(4):401-44.

2. Glen CM, Kemp ML, Voit EO. Agent-based modeling of morphogenetic systems: advantages and challenges. PLoS Comput Biol. 2019;15(3):1006577.

3. Fletcher AG, Cooper F, Baker RE. Mechanocellular models of epithelial morphogenesis. Philos Trans R Soc B Biol Sci. 2017;372(1720):20150519.

4. Rejniak KA, Anderson ARA. Hybrid models of tumor growth. Wiley Interdiscip Rev Syst Biol Med. 2010;3(1):115-25. https://doi.org/10.1002/wsbm.102.

5. Metzcar J, Wang Y, Heiland R, Macklin P. A review of cell-based computational modeling in cancer biology. JCO Clin Cancer Inform. 2019;2:1-13.

6. Peirce SM, Van Gieson EJ, Skalak TC. Multicellular simulation predicts microvascular patterning and in silico tissue assembly. FASEB J. 2004;18(6):731-3.

7. Graner F, Glazier JA. Simulation of biological cell sorting using a two-dimensional extended potts model. Phys Rev Lett. 1992;69(13):2013.

8. Meineke FA, Potten CS, Loeffler M. Cell migration and organization in the intestinal crypt using a lattice-free model. Cell Prolif. 2001:34(4):253-66. https://doi.org/10.1046/j.0960-7722.2001.00216.x.

9. Drasdo D, Hoehme S. A single-cell-based model of tumor growth in vitro: monolayers and spheroids. Phys Biol. 2005:2(3):133-47. https://doi.org/10.1088/1478-3975/2/3/001

10. Fletcher AG, Osterfield M, Baker RE, Shvartsman SY. Vertex models of epithelial morphogenesis. Biophys J. 2014;106(11):2291-304.

11. Rejniak KA. An immersed boundary framework for modelling the growth of individual cells: an application to the early tumour development. J Theor Biol. 2007;247(1):186-204.

12. Newman TJ. Modeling multicellular structures using the subcellular element model. In: Anderson ARA, Chaplain MAJ, Rejniak KA, editors. Single-cell-based models in biology and medicine. mathematics and biosciences in interaction. Birkhäuser: Basel; 2007. p. 221-39.

13. Osborne JM, Fletcher AG, Pitt-Francis JM, Maini PK, Gavaghan DJ. Comparing individual-based approaches to modelling the self-organization of multicellular tissues. PLoS Comput Biol. 2017;13(2):1005387. https://doi.org/10.1371/ journal.pcbi.1005387.

14. Cooper FR, Baker RE, Bernabeu MO, Bordas R, Bowler L, Bueno-Orovio A, Byrne HM, Carapella V, Cardone-Noott L, Cooper J, et al. Chaste: cancer, heart and soft tissue environment. J Open Sour Softw. 2020;5(47):1848.

15. Delile J, Herrmann M, Peyriéras N, Doursat R. A cell-based computational model of early embryogenesis coupling mechanical behaviour and gene regulation. Nat Commun. 2017;8:13929. https://doi.org/10.1038/ncomms13929. 
16. Ghaffarizadeh A, Heiland R, Friedman SH, Mumenthaler SM, Macklin P. PhysiCell: an open source physics-based cell simulator for 3-d multicellular systems. PLoS Comput Biol. 2018;14(2):1005991. https://doi.org/10.1371/journal.pcbi. 1005991.

17. Germann P, Marin-Riera M, Sharpe J. yal||a: GPU-powered spheroid models for mesenchyme and epithelium. Cell Syst. 2019;8(3):261-6.

18. Hoehme S, Drasdo D. A cell-based simulation software for multi-cellular systems. Bioinformatics. 2010;26(20):26412. https://doi.org/10.1093/bioinformatics/btq437.

19. Sütterlin T, Kolb C, Dickhaus H, Jäger D, Grabe N. Bridging the scales: semantic integration of quantitative sbml in graphical multi-cellular models and simulations with episim and copasi. Bioinformatics. 2013;29(2):223-9.

20. Kang S, Kahan S, McDermott J, Flann N, Shmulevich I. Biocellion: accelerating computer simulation of multicellular biological system models. Bioinformatics. 2014;30(21):3101-8. https://doi.org/10.1093/bioinformatics/btu498.

21. Griffiths DF, Higham DJ. Numerical methods for ordinary differential equations: initial value problems. London: Springer; 2010.

22. Chapra SC. Applied numerical methods with matlab for engineers and scientists. New York: McGraw-Hill; 2012.

23. Fletcher AG, Osborne JM. Seven challenges in the multiscale modeling of multicellular tissues. In: WIREs mechanisms of disease, 2020. p. 1527

24. Kursawe J, Baker RE, Fletcher AG. Impact of implementation choices on quantitative predictions of cell-based computational models. J Comput Phys. 2017;345:752-67.

25. Pathmanathan P, Cooper J, Fletcher A, Mirams G, Murray P, Osborne J, Pitt-Francis J, Walter A, Chapman SJ. A computational study of discrete mechanical tissue models. Phys Biol. 2009;6(3):036001. https://doi.org/10.1088/14783975/6/3/036001.

26. Atwell $\mathrm{K}$. Investigating the interplay between cellular mechanics and decision-making in the c. elegans germ line. $\mathrm{PhD}$ thesis, University of Oxford, 2016.

27. Mathias S, Coulier A, Bouchnita A, Hellander A. Impact of force function formulations on the numerical simulation of centre-based models. Bull Math Biol. 2020. https://doi.org/10.1007/s11538-020-00810-2.

28. Walt S, Colbert SC, Varoquaux G. The numpy array: a structure for efficient numerical computation. Comput Sci Eng. 2011;13(2):22-30.

29. Okuta R, Unno Y, Nishino D, Hido S, Loomis C. Cupy: a numpy-compatible library for nvidia gpu calculations. In: Proceedings of workshop on machine learning systems (LearningSys) in the thirty-first annual conference on neural information processing systems (NIPS), 2017.

30. Mathias S, Coulier A. CBMOS Github page, 2021. https://github.com/somathias/cbmos. Accessed 26 Aug 2021.

31. Mathias S, Coulier A. CBMOS documentation page, 2021. https://somathias.github.io/cbmos/. Accessed 26 Aug 2021.

32. Bisong E. Google colaboratory. In: Building machine learning and deep learning models on google cloud platform: a comprehensive guide for beginners. Berkeley: Apress; 2019. p. 59-64. https://doi.org/10.1007/978-1-4842-4470-8_7.

33. Google: Google Colaboratory. https://research.google.com/colaboratory/. Accessed 26 Aug 2021.

34. Drasdo D. Buckling instabilities of one-layered growing tissues. Phys Rev Lett. 2000;84(18):4244-7. https://doi.org/ 10.1103/physrevlett.84.4244.

35. Drasdo D, Loeffler M. Individual-based models to growth and folding in one-layered tissues: intestinal crypts and early development. Nonlinear Anal Theory Methods Appl. 2001;47(1):245-56. https://doi.org/10.1016/s0362546x(01)00173-0.

36. Schaller G, Meyer-Hermann M. Multicellular tumor spheroid in an off-lattice Voronoi-Delaunay cell model. Phys Rev E. 2005. https://doi.org/10.1103/physreve.71.051910.

37. Galle J, Loeffler M, Drasdo D. Modeling the effect of deregulated proliferation and apoptosis on the growth dynamics of epithelial cell populations in vitro. Biophys J. 2005;88(1):62-75.

38. Purcell EM. Life at low Reynolds number. Am J Phys. 1977:45(1):3-11. https://doi.org/10.1119/1.10903.

39. Hindmarsh AC. Odepack, a systematized collection of ode solvers. IMACS Trans Sci Comput. 1983;1:55-64.

40. Harvey DG, Fletcher AG, Osborne JM, Pitt-Francis J. A parallel implementation of an off-lattice individual-based model of multicellular populations. Comput Phys Commun. 2015;192:130-7. https://doi.org/10.1016/j.cpc.2015.03. 005.

41. Millman KJ, Pérez F, Stodden V, Leisch F, Peng RD. Developing open-source scientific practice. In: Implementing reproducible research, 2014. p. 149.

42. Hockney RW, Eastwood JW. Computer simulation using particles. Boca Raton: CRC Press; 1988.

43. Harris CR, Millman KJ, van der Walt SJ, Gommers R, Virtanen P, Cournapeau D, Wieser E, Taylor J, Berg S, Smith NJ, Kern R, Picus M, Hoyer S, van Kerkwijk MH, Brett M, Haldane A, del Río JF, Wiebe M, Peterson P, Gérard-Marchant P, Sheppard K, Reddy T, Weckesser W, Abbasi H, Gohlke C, Oliphant TE. Array programming with NumPy. Nature. 2020;585(7825):357-62. https://doi.org/10.1038/s41586-020-2649-2.

44. Dask Development Team: Dask: library for dynamic task scheduling, 2016. https://dask.org.

45. Abbasi H. Sparse: a more modern sparse array library. In: Proceedings of the 17 th python in science conference, 2018. p. 27-30.

46. Distributed (Deep) Machine Learning Community: MinPy, 2016. https://github.com/dmlc/minpy. Accessed 26 Aug 2021.

47. Chen T, Li M, Li Y, Lin M, Wang N, Wang M, Xiao T, Xu B, Zhang C, Zhang Z. Mxnet: a flexible and efficient machine learning library for heterogeneous distributed systems. In: Workshop on machine learning systems, 2015.

48. Maia FRNC. afnumpy: a GPU-ready drop-in replacement for NumPy, 2017. https://doi.org/10.5281/zenodo.262201. Accessed 26 Aug 2021.

49. Nickolls J, Buck I, Garland M, Skadron K. Scalable parallel programming with cuda: is cuda the parallel programming model that application developers have been waiting for? Queue. 2008:6(2):40-53.

50. Mathias S, Coulier A. CBMOS page on the python package index, 2021. https://pypi.org/project/cbmos/. Accessed 26 Aug 2021 
51. Hairer E, Wanner G. Solving ordinary differential equations II: stiff and differential-algebraic problems. Berlin: Springer; 1996.

52. Saad Y, Schultz MH. GMRES: a generalized minimal residual algorithm for solving nonsymmetric linear systems. SIAM J Sci Comput. 1986;7:856-69.

53. Knoll DA, Keyes DE. Jacobian-free Newton-Krylov methods: a survey of approaches and applications. J Comput Phys. 2004;193:357-97.

54. Kluyver T, Ragan-Kelley B, Pérez F, Granger BE, Bussonnier M, Frederic J, Kelley K, Hamrick JB, Grout J, Corlay S, et al. Jupyter notebooks - a publishing format for reproducible computational workflows, 2016.

55. Creative commons: creative commons - attribution 4.0 international-CC BY 4.0, 2022. https://creativecommons. org/licenses/by/4.0/. Accessed 06 Jan 2022.

\section{Publisher's Note}

Springer Nature remains neutral with regard to jurisdictional claims in published maps and institutional affiliations.

Ready to submit your research? Choose BMC and benefit from:

- fast, convenient online submission

- thorough peer review by experienced researchers in your field

- rapid publication on acceptance

- support for research data, including large and complex data types

- gold Open Access which fosters wider collaboration and increased citations

- maximum visibility for your research: over 100M website views per year

At BMC, research is always in progress.

Learn more biomedcentral.com/submissions 